\title{
Experimental Study on the Sensitive Emission Lines Intensities of Metal Samples Using Laser Ablation Technique and Its Comparison to Arc Discharge Technique*
}

\author{
Eko Susilowati ${ }^{1}$, Tjung $\mathrm{Jie} \mathrm{Lie}^{2}$ \& Hendrik Kurniawan ${ }^{2}$ \\ ${ }^{1}$ Department of Mathematics and Natural Sciences Education, Faculty of Education, \\ Lambung Mangkurat University, H. Hasan Basry, P.O. Box 87, \\ Banjarmasin 70123, Indonesia. \\ ${ }^{2}$ Research Center of Maju Makmur Mandiri, 40 Srengseng Raya, Kembangan, Jakarta \\ Barat 11630, Indonesia. E-mail: kurnia18@cbn.net.id
}

\begin{abstract}
An experimental study has been carried out to measure the sensitive emission lines intensities of several metal samples (copper, zinc, silver, gold, gallium, nickel, silicone and iron) using laser ablation technique conducted in low pressure surrounding gas by means of Laser Induced Shock Wave Plasma Spectroscopy (LISPS) and in atmospheric pressure region using Laser Induced Breakdown Spectroscopy (LIBS). In both cases the Nd-YAG laser was operated at its fundamental wavelength of 1,064 nm with pulse duration of $8 \mathrm{~ns}$ and its intensity tightly focused on the metal samples in helium or air as an ambient gas. The laser energy was fixed at approximately $100 \mathrm{~mJ}$ using a set of neutral density filters placed tilted in front of the laser output window. The result of the intensity measurements showed a good agreement which those obtained using arc discharge technique as shown in Massachusetts Institute of Technology Wavelength Table. Further evaluation of these results on the basis of standard deviation leads to the conclusion that LISPS is more favorable for quantitative analysis compared to LIBS. It was further shown that replacing air by helium gas at low pressure improve to some extent the LISPS reproducibility and sensitivity.
\end{abstract}

Keywords: arc discharge; laser-induced breakdown spectroscopy; laser-induced shock wave plasma spectroscopy; metal samples; sensitive emission lines intensities.

\section{Introduction}

Atomic Emission Spectroscopy gained unprecedented recognition shortly after the discovery of spark discharge and arc discharge techniques. These two techniques, along with the "discharge tube" technique, have yielded many

\footnotetext{
* author to whom correspondence should be sent: Hendrik Kurniawan, Research Center of Maju Makmur Mandiri, 40 Srengseng Raya, Kembangan, RT002/RW06, Jakarta Barat 11630, Tel: 62-21-5867663, 5867660, 5867601, Fax: 62-21-5867670, 5809144, E-mail: kurnia18@cbn.net.id, http://www.mmm.or.id
} 
benefits in the area of elemental analysis both qualitatively and quantitatively. From 1900s to the 1930s, the Massachusetts Institute of Technology undertook the massive task of mapping more than 100,000 atomic emission lines of elements which could be excited under normal excitation conditions. Compiled by Harrison et $\mathrm{al}^{1}$, this book consists of all the wavelengths of all the elements ranging from 2,000 A to 10,000 A, with an accuracy of $0.001 \mathrm{~A}$. This book, now known as the M.I.T Wavelength Tables I, was later further improved by Frederick M Phelps III $^{2}$ who added the atomic emission lines in a vacuum, calculated using Edlen's dispersion theorem, allowing the conversion from wavelengths in air to wavelengths in a vacuum. This monumental work is now known as the M.I.T Wavelength Tables II.

After the discovery of laser by Maiman, the method of atomic spectroscopy grew rapidly with the use of high-powered lasers with short pulse duration. This revolutionary technique was highly favored in comparison with the spark discharge, arc discharge and discharge tube techniques as the atomic emission obtained was relatively free from electrode emission, commonly containing carbon. Along with the development of laser spectroscopy, a new term was coined specifically for this emission analysis technique, nemely the Laser Atomic Emission Spectrochemical Analysis, more widely known as LAESA, first published by Brech et $\mathrm{al}^{3}$ in 1962.

The recent development in laser ablation emission spectrometric analysis $(\text { LAESA })^{4-5}$ can be categorized more or less into two main streams. One of these employs a high pressure surrounding gas, as originally developed by Radziemski et $\mathrm{al}^{7-12}$ and is usually called laser induced breakdown spectroscopy (LIBS) in connection with the breakdown mechanism in the gas. In this method, a high peak power laser with short duration such as a pulsed Nd-YAG laser is focused onto a sample at atmospheric pressure. In order to remove the interfering background from the intense continuous spectrum emitted by the high density plasma, a gated OMA unit is incorporated into the detection system. The development of LAESA method along the other direction involves the use of low gas pressures. With the laser plasma produced under a reduced gas pressure, the intensity of background emission becomes considerably reduced. ${ }^{13-20}$ In connection with this second line of development, we have shown in a series of experiments that a laser-induced shock wave plasma was generated when a pulsed gas laser, such as an $\mathrm{N}_{2}$ laser, a TEA $\mathrm{CO}_{2}$ laser, or an excimer laser, was focused onto a solid target at reduced gas pressures around 1 Torr. ${ }^{21-36}$ It was found in each case that the laser plasma invariably consisted of two distinct parts. The first part occupied a small region of high temperature (the primary plasma), which gave rise to an intense and continuous emission for a short time, just above the surface of the target. The second part (the secondary plasma) expanded with time around the primary plasma emitting sharp atomic 
spectral lines with negligibly low background emission. By means of timeresolved measurements in our experiments using a TEA $\mathrm{CO}_{2}$ laser ${ }^{22}$ and an excimer laser, ${ }^{23}$ we were able to demonstrate that atoms in this secondary plasma were excited by the shock wave, while the primary plasma acted as a source of explosion energy source. We have referred to this method as LaserInduced Shock Wave Plasma Spectroscopy (LISPS).

The present work was carried out in order to elucidate whether the intensities of sensitive emission lines of several metal samples obtained by arc discharge as described in M.I.T. Wavelength Tables could be used as the same map for laser technique. To that end, emission intensities measurements were carried out with LISPS in air at 5 Torr and in helium at 35 Torr and by LIBS in air at 760 Torr, and the results were analyzed in comparison with those obtained by arc discharge. Further data regarding reproducibility of the emission intensities produced by the above technique was also conducted in the hope to obtain the best laser ablation technique for spectrochemical application.

\section{Basic Physical Mechanism of Laser-Induced Plasma}

\subsection{Laser Induced Breakdown Spectroscopy}

Soon after the development of ruby laser, experiments were carried out by bringing a laser beam of sufficiently high power to a sharp focus in atmospheric air, where a bright spark was produced at the focus accompanied by a loud click. It is in many ways similar to a breakdown phenomena induced by arc discharge or intense microwave radiation. The essential feature is that the gas, which is normally transparent and non-conducting, becomes ionized by the laser light, and becomes highly absorptive for the laser beam. Consequently rapid local heating occurs, leading to the production of dense, strongly ionized plasma. Since then, numerous experiments have been carried out on various gases by means of different kinds of pulse laser available, such as ruby laser, Nd-glass laser, Nd-YAG laser and $\mathrm{CO}_{2}$ laser. Many reviews on the plasma generation in high pressure buffer gas have been published ${ }^{37, a-40}$ and the related theoretical description has also been successfully developed.

The starting point of laser-induced plasma in gas at atmospheric pressure is the gas breakdown in the focal region. As soon as the gas breakdown occurs, the ionized region begins to absorb strongly the energy from the remainder of laser pulse. This process causes rapid heating of the ionized gas, and thus generating high temperature gas plasma. The two main mechanisms responsible for initiating the gas breakdown are the multi-photon ionization at the beginning of laser irradiation and inverse-bremsstrahlung process after free electrons were produced. 
Following the breakdown of the gas, the luminous plasma starts to expand outward in all direction from the focal volume. Rapid expansion of the heated gas can produce a shock wave, which resembles a detonation wave if the shock front absorbs the laser light strongly. A plasma temperature (ion temperature) as high as $2.5 \times 10^{5} \mathrm{~K}$ can be achieved shortly after the laser pulse reaches its maximum in the case of a Q-switch ruby laser with an output power of $25 \mathrm{MW}$ focused in air of $1 \mathrm{~atm} .^{38}$ The plasma generated by the laser pulse emits intense light over a broad spectrum, mainly in the ultra violet region. Some of this radiation may produce ionization on being absorbed by the cooler surrounding gas, which will in turn absorb incoming laser light more strongly.

Heating of solids by laser pulses, even as short as Q-switch pulses, is essentially thermal in nature. A quantum of optical energy is absorbed by an electron, which is thus raised to a higher energy state in the conduction band. The excited electrons collide with other electrons as well as phonons and thereby give up their energy. These are the same collision processes which govern the transfer of heat. The mean free time between collisions of electrons in conductor is of the order of $10^{-12}$ to $10^{-13}$ seconds, which is a very short period of time compared to the pulse duration of the Q-switch laser, which is in the order of $10^{-8}$ seconds. Within the duration of a laser pulse, the electrons, after absorbing the photon, will undergo many collisions and the energy absorbed will be distributed and passed on to the lattice, resulting in effective and instantaneous energy transferred to the solid as a heat. The absorption coefficient for metals is high enough $\left(10^{5}-10^{6} \mathrm{~cm}^{-1}\right)$ that the laser energy can be considered to be deposited at the surface initially, followed by its transfer into the metal by conduction.

In general, the amount of mass removed from the surface by Q-switch laser bombardment is less than that due to normal laser bombardment. The crater depth caused by Q-switch pulse lasers are relatively shallow, in the order of 10 to $100 \mu \mathrm{m}$, while the depth could reach $900 \mu \mathrm{m}$ in the case of normal oscillation pulse laser. The other important difference is that, in the case of normal oscillation pulse laser, much of the material removed is in the liquid phase rather than the vapor phase, which can be observed in the crater wall on the surface, whereas in the case of Q-switch pulse laser, most of the material removed is in the vapor state. Vaporization by the normal oscillation pulse laser in the range of irradiance $10^{6}-10^{7} \mathrm{~W} \mathrm{~cm}^{-2}$ can be regarded as ordinary evaporation at the normal boiling point, with continuous mass removal from the surface during the irradiation. The observed velocities of vaporized material are on the order of $10^{4} \mathrm{~cm} \mathrm{~s}^{-1}$, consistent with velocities of atoms at normal boiling temperatures. With the Q-switch pulse laser at irradiance in excess of $10^{8} \mathrm{Wcm}^{-2}$ , vaporization takes place at temperature above the normal vaporization temperature, and atoms are emitted from the surface at higher velocities, 
around $10^{6} \mathrm{~cm} \mathrm{~s}^{-1}$ although the amount vaporized is smaller. This phenomenon cannot be explained simply by heat conduction. A number of models have been proposed to explain this phenomenon as documented by Hughes. ${ }^{38,41-48}$

\subsection{Laser Induced Shock Wave Plasma Spectroscopy}

In the previous studies of the plasma generation under reduced pressure, ${ }^{21-36}$ we have shown that a plasma having characteristics favorable to spectrochemical analysis could actually be generated by using a pulsed gas laser with short duration, such as the nitrogen laser, ${ }^{21-22}$ carbon dioxide laser, ${ }^{23,25-27}$ and excimer laser, ${ }^{24}$ when the pressure of the surrounding gas was reduced to around 1 Torr. In these cases, the plasma invariably consists of two distinct parts. The first part, which is called the primary plasma, occupies a small area and gives off intense continuous emission spectra for a short time just above the surface of the target. The other part, called the secondary plasma, expands with time around the primary plasma with near hemispherical shape, emitting sharp atomic spectral lines with negligibly low background.

In a series of subsequent time-resolved experiments using a carbon-dioxide laser, ${ }^{49-50}$ excimer laser, ${ }^{28}$ and Nd-YAG laser, ${ }^{31-34}$ we demonstrated that this secondary plasma was excited by the shock-wave, while the primary plasma acted as an initial explosion energy source. We have offered a theory with respect to the excitation mechanism of the secondary plasma as follows. Right after the cessation of the primary plasma, atoms gush out from the primary plasma at supersonic speed. It is assumed that the surrounding gas plays the role of damping material, impeding the free expansion of the propelling atoms, by forming something like a wall against which compression is taking place. As a result of this compression, a blast wave is generated in the surrounding gas. The most important point of the shock wave model is that the energy required to produce the secondary plasma is supplied in the form of kinetic energy from the propelling atoms. By means of this compression, the kinetic energy of the propelling atoms is converted into thermal energy in the plasma, by which atoms are excited.

In a different experiments carried out recently, ${ }^{36}$ we proved that at the initial stage of the secondary plasma expansion, the front of the propelling atoms and the front of the blast wave coincided and moved together with time. However, at a later stage, the front of the atom cluster was separated from that of the blast wave induced in the surrounding gas. In order to further explain the characteristics of the secondary plasma, we presented a hypothetical model, in which the dynamical process of the secondary plasma was separated into two stages, namely, the shock excitation stage which was followed by the cooling stage. Thus in the shock excitation region, atoms were excited by the heat arising from strong compression between the shock front and propelling atoms. 
The cluster of propelling atoms was supposed to move in a manner somewhat like a piston pushing the surrounding gas. We called this stage the shock excitation stage. Soon afterward, the cluster of atoms began to slow down while loosing its energy to the surrounding gas. As a result, no further excitation took place, since the compression between the shock front and propelling atoms could no longer be sustained due to the widening separation between them, while the cluster of atoms continued to move forward with its residual momentum. Since the surrounding gas behind the shock wave was left with high temperature, and the secondary plasma also contained a large amount of residual thermal energy, the cooling of the plasma was bound to proceed slowly with time. We have called this part of the process the cooling stage.

Sedov $^{51}$ has derived a theoretical relationship between the propagation time $(\mathrm{t})$ of the blast wave and the distance of its front, $r$ from the position of the source of explosion as follows:

$$
r=(E o / \alpha \rho)^{1 / 5} t^{2 / 5}
$$

where Eo is the initial explosion energy, $\rho$ is the density of the surrounding gas and $\alpha$ is the constant involving $\gamma$ (the ratio of specific heats of the gas). Also it is well known that for detonation wave, $r$ is proportional to $t^{3 / 5}$. In the case of LISPS, we have proved the shock front position is proportional to the initial explosion energy $\left(\mathrm{E}_{0}{ }^{1 / 5}\right)$ whereas the shock front position was also proportional to $\mathrm{t}^{2 / 5}$ which is in good agreement with Sedov equation.

\section{Experimental Procedure}

The basic experimental setup used in this research is similar to the one used in our previous work ${ }^{28}$ except a change in the laser source. In this experiment, the laser radiation from a 1,064 nm Nd-YAG (Quanta Ray, GCR-12S, $400 \mathrm{~mJ}, 8$ ns) is operated in a Q-sw mode at $5 \mathrm{~Hz}$ repetition rate with the laser output energy fixed at $100 \mathrm{~mJ}$ by means of a set of neutral density filters. The laser beam was focused by a multilayer lens $(f=100 \mathrm{~mm})$ through a BK-7 window onto the surface of the sample. The shot-to-shot fluctuation of the laser was estimated to be approximately $3 \%$.

The samples employed in this experiments, consist of $\mathrm{Cu}, \mathrm{Zn}, \mathrm{Ni}, \mathrm{Ga}, \mathrm{Ag}, \mathrm{Si}, \mathrm{Fe}$ and $\mathrm{Au}$ (Rare Metallic Co, 4N) In each experiment, the sample is placed in a small, vacuum-tight metal chamber measuring $11 \mathrm{~cm} \times 11 \mathrm{~cm} \times 12.5 \mathrm{~cm}$, which could be evacuated with a vacuum pump and filled with surrounding gas at desired pressure. The gas flow through the chamber is regulated by a needle valve in the air line and another valve in the pumping line. The chamber pressure is measured and monitored by means of a digital absolute vacuummeter. The sample, together with the whole chamber and multi layer lens, can 
be moved with the aid of a step motor for movement in the laser beam direction and a micrometer for movement perpendicular to the laser beam direction.

The plasma radiation is detected by an optical multichannel analyzer (OMA system, Princeton Instrument IRY-700) attached to a monochromator with a focal length of $150 \mathrm{~mm}$ and connected to an optical fiber with its entrance placed in front of the observation window of the vacuum chamber. The detector of this measuring system is a gateable intensified photodiode array with gating width ranging from $40 \mathrm{~ns}$ to $80 \mathrm{~ms}$. The spectral window covered by the detector has a width of $80 \mathrm{~nm}$ at $500 \mathrm{~nm}$. The detected signals are monitored on a screen.

\section{$4 \quad$ Results and Discussion}

As we have reported in our previous works, ${ }^{21-28}$ the secondary plasma generated at around 5 Torr could be easily distinguished from the primary plasma even by naked eyes. The secondary plasma generally displays a characteristic symmetrical shape with brilliant color due to emission from the constituent atoms, while the primary plasma displays a dense white color associated with the continuum emission. The separation between the secondary plasma and primary plasma is clearly visible for pressure up to 300 Torr. Above 300 Torr,

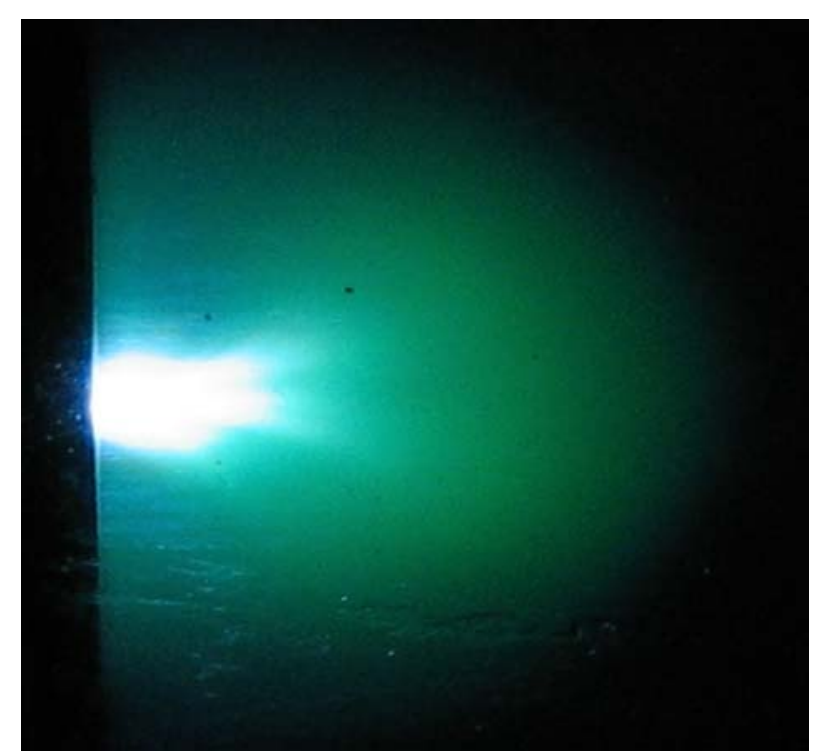

Figure 1(a) Cu plasma generated under surrounding air pressure of 5 Torr. The laser energy of 1,064 nm Nd-YAG laser was fixed at $100 \mathrm{~mJ}$. The diameter of the plasma was around $30 \mathrm{~mm}$. 
the plasma as a whole appears like a primary plasma emitting only strong and dense white light visible to the naked eyes. The secondary plasma produced from the copper sample gives rise to a bright green color, which mainly arises from the $\mathrm{Cu}$ I $521.8 \mathrm{~nm}$, Cu I $515.3 \mathrm{~nm}$ and $\mathrm{Cu}$ I $510.5 \mathrm{~nm}$ emission lines. The shape of the secondary plasma is hemispherical, and the radius increases with decreasing pressure of the surrounding gas. When the pressure is reduced to 0.5 Torr however, the presence of the secondary plasma becomes rather vague. It is therefore important to examine the plasma characteristics at this surrounding gas pressure in order to ascertain the origin of this change. Fig. 1 shows the copper plasma generated under (a) air pressure of 5 Torr and (b) helium pressure of 35 Torr and (c) air pressure of 760 Torr. It should be noted that even the feature of Fig. 1(a) and Fig. 1(b) is quite similar, once should noteworthy that the continuum emission in the case of air at 5 Torr which is represented by the white dense color is much stronger in the case of air than that of helium. This feature is closely related to the improvement of signal to background ratio in the case of helium plasma. We shall return to this point after we presented the spectra of the above plasma. In contrast to Fig. 1(a) and Fig. 1(b), no secondary plasma was observed when the air pressure is increased all the way up to 760 Torr. We should note that the LIBS plasma is mainly a high density and high temperature plasma featuring strong continuum emission.

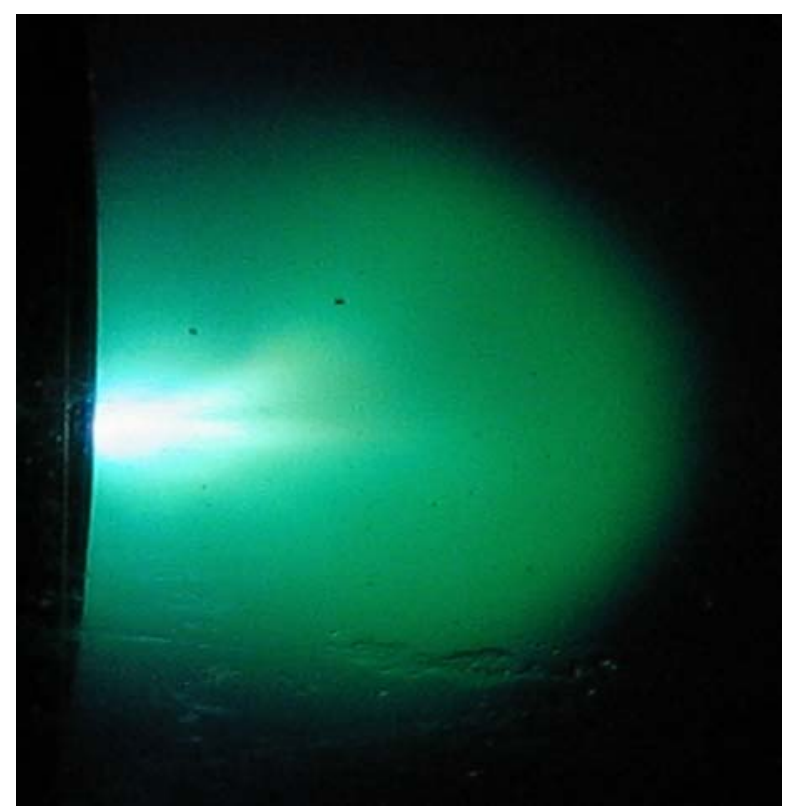

Figure 1(b) Cu plasma generated under surrounding helium pressure of 35 Torr. The laser energy of 1,064 nm Nd-YAG laser was fixed at $100 \mathrm{~mJ}$. The diameter of the plasma was around $30 \mathrm{~mm}$. 


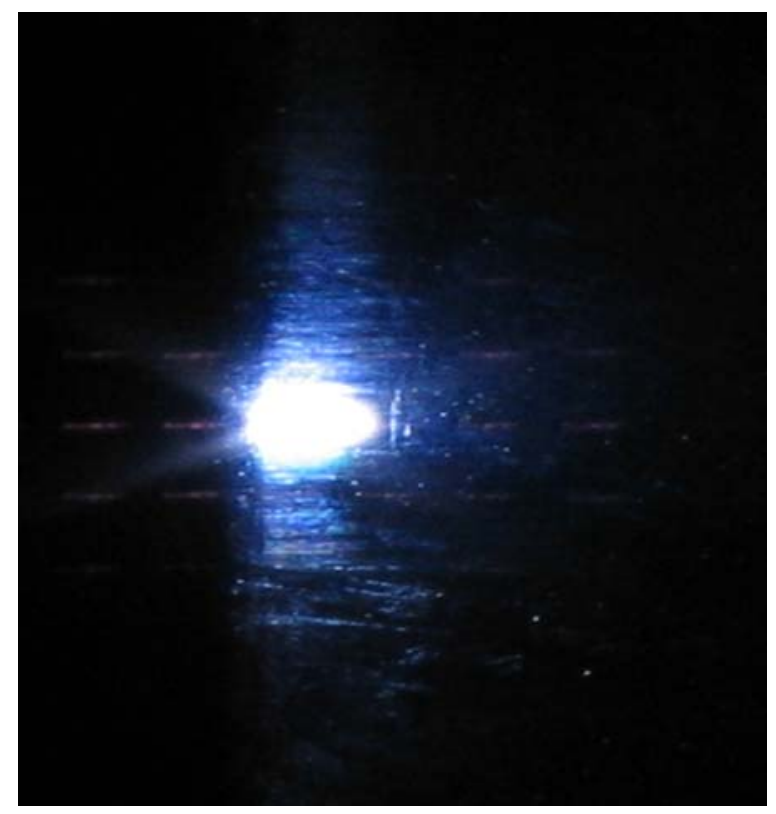

Figure 1(c) Cu plasma generated under surrounding air pressure of 760 Torr. The laser energy of 1,064 nm Nd-YAG laser was fixed at $100 \mathrm{~mJ}$. The diameter of the plasma was around $4 \mathrm{~mm}$.

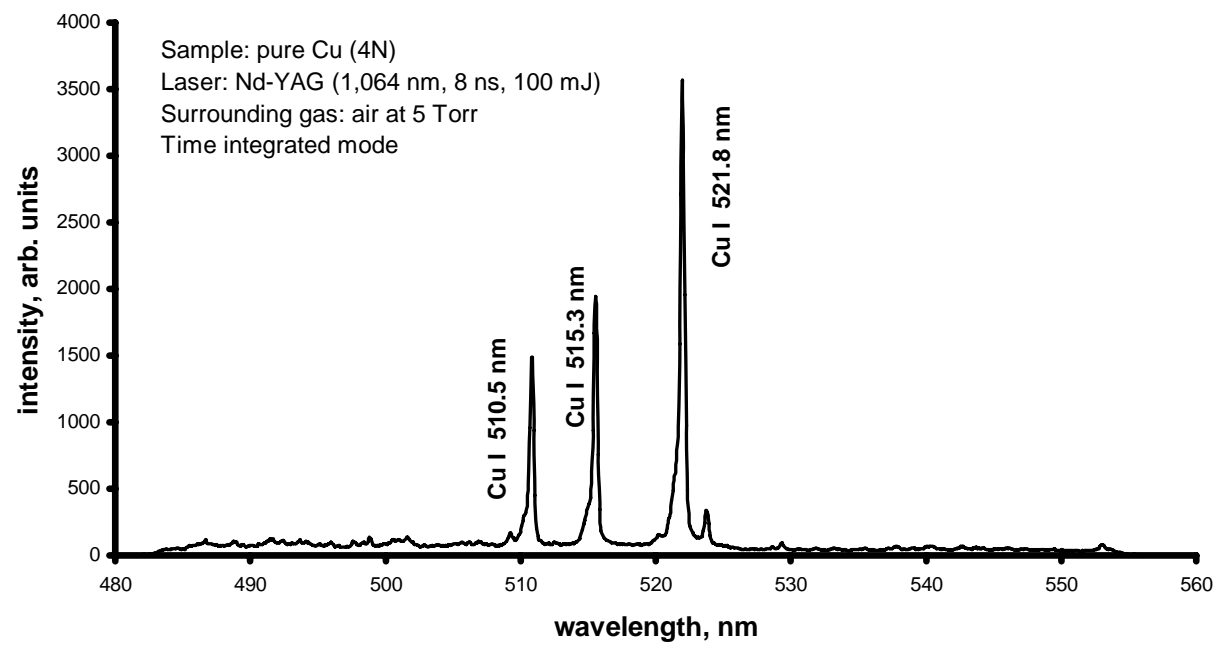

Figure 2(a) Timely and spatially integrated emission spectra of $\mathrm{Cu}$ plasma generated in air at 5 Torr (LISPS) using $100 \mathrm{~mJ}$ Nd-YAG laser irradiation. 


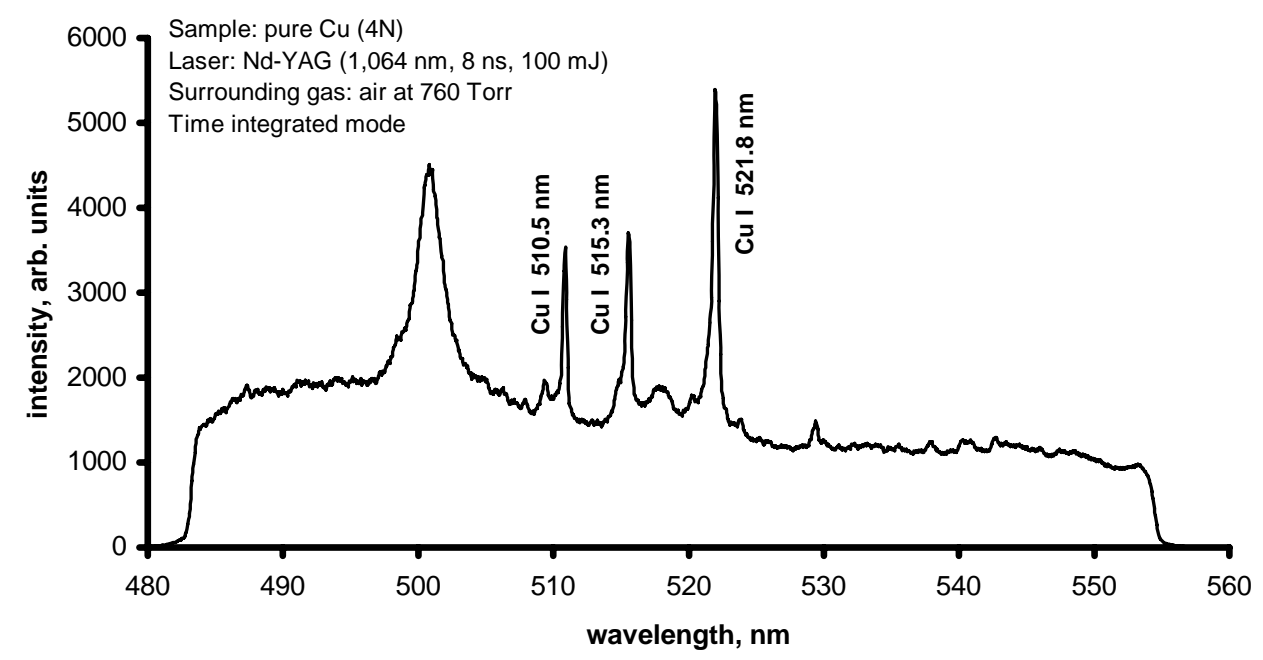

Figure 2(b) Timely and spatially integrated emission spectra of $\mathrm{Cu}$ plasma generated in air at 760 Torr (LIBS) using $100 \mathrm{~mJ}$ Nd-YAG laser irradiation.

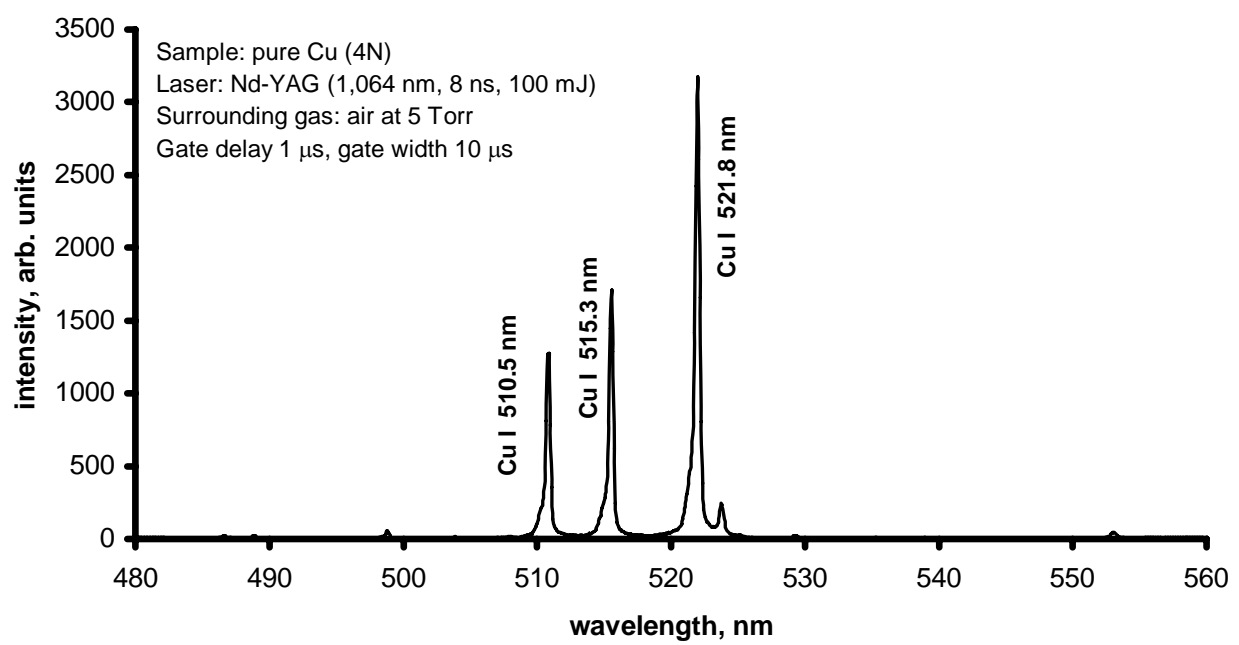

Figure 3(a) Spatially integrated and time resolved emission spectra of $\mathrm{Cu}$ plasma generated in air at 5 Torr (LISPS) using $100 \mathrm{~mJ}$ Nd-YAG laser irradiation.

Fig. 2(a) shows the temporally and spatially integrated emission spectra of the secondary plasma of a copper sample under air pressure of 5 Torr (a) and under air pressure of 760 Torr (b).It is clearly seen that in the case of LIBS without 
using gating technique, the signal to background ratio is extremely bad as compared in the case of air at 5 Torr. This discrepancy lead to unfavorable characteristics of LIBS due to high cost gated detector system. Even if the gated system was employed, the signal to background ratio (S/B) in the case of LIBS remained much lower than that in LISPS. The improvement of S/B by gated detector is more pronounced in the case of LISPS by comparing Fig. 3(a) and Fig. 3(b). In addition to higher background, one also observed the broadening of the emission lines. This broadening effect has been explained in term of Stark effect in the case of 760 Torr surrounding air pressure shown in Fig. 3(b).

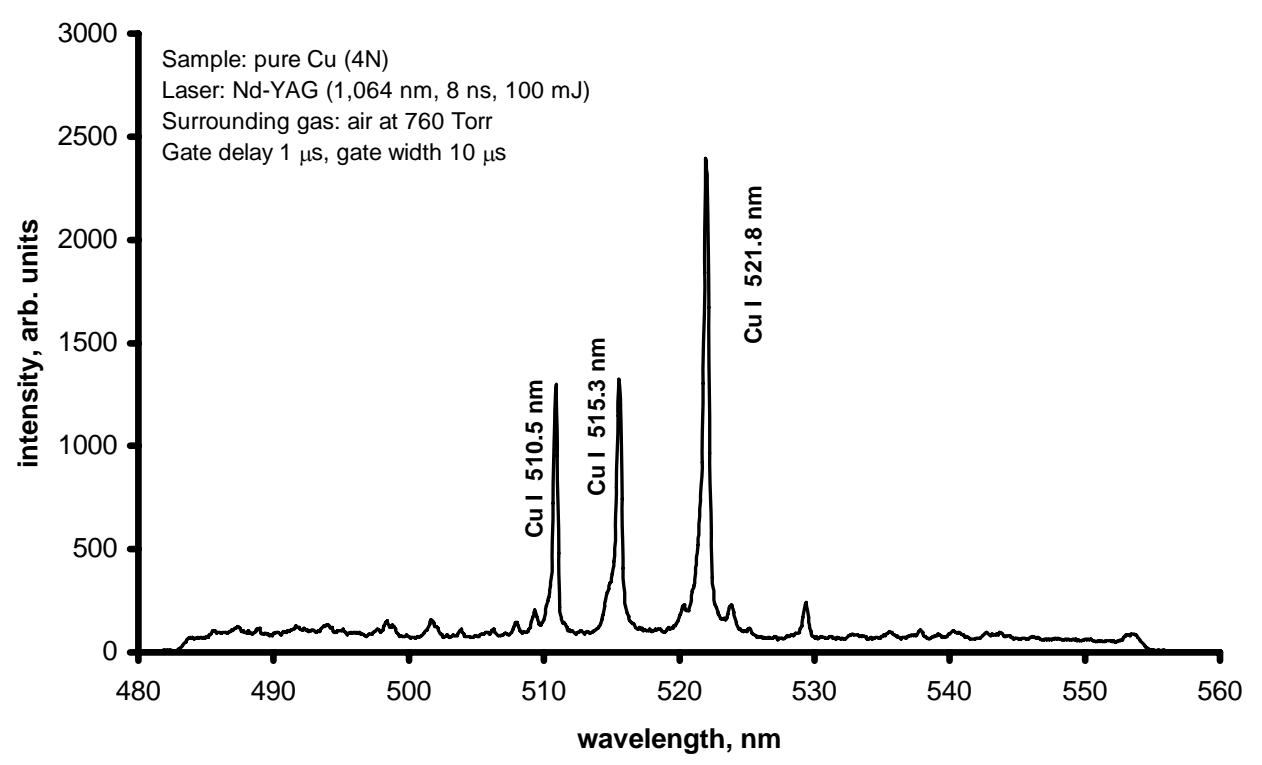

Figure 3(b) Spatially integrated and time resolved emission spectra of $\mathrm{Cu}$ plasma generated in air at 760 Torr (LIBS) using $100 \mathrm{~mJ}$ Nd-YAG laser irradiation.

One should note that in the case of gated detection system, the background signal of LISPS is close to zero which will result in an extremely low detection limit. We further studied the effect of using helium gas in place of air, and the result is presented in Fig. 4 for spatially-integrated time- resolved spectra of $\mathrm{Cu}$ plasma.

The result obtained in Fig. 4 share exactly the same feature of extremely low background in Fig. 3(a). Clearly Fig. 4 helps to explain the difference between the plasma features described in Fig. 1(a) and 1(b). Compared to Fig. 3(a), the 
use of helium gas brings about a significant increase in the emission intensity which will in turn lower the detection limit.

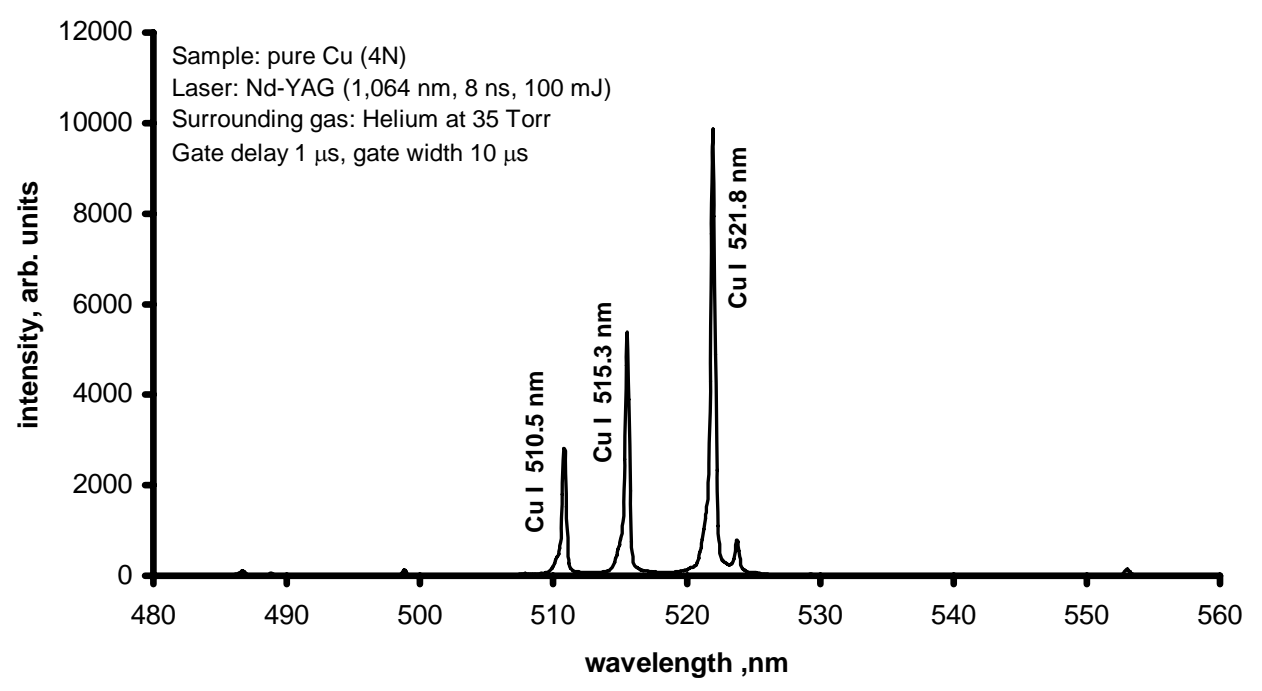

Figure 4 Spatially integrated and time resolved emission spectra of Cu plasma generated in helium at 35 Torr (LISPS) using $100 \mathrm{~mJ}$ Nd-YAG laser irradiation.

As explained in the Introduction and Basic Theory sections, laser target interaction can be carried out under buffer gas pressure of 760 Torr, more widely known as the LIBS method; or under reduced gas pressure of $1-10$ Torr, using a method known as LISPS, recently developed by Kagawa et al. The existence of excitation and cooling states, allows for the emission lines to be selected accurately, with careful consideration given to the laser wavelength, laser pulse width, type of target and type of gas being used. In this study, measurements using the LISPS method uses air pressurized at 5 Torr and helium at 35 Torr. The different pressure of helium is chosen to provide a different surrounding gas of approximately the same density (atomic mass of helium (4) at 35 Torr is $1 / 7$ of molecular mass of air (28.8) at 5 Torr) since the gas density plays a crucial role in LISPS according to Eq. 1.

In order to clarify the excitation mechanism of the secondary plasma, it is also very useful to know the averaged temperature of the secondary plasma. The plasma temperature was measured by the Boltzmann two-line method, assuming validity of the Boltzmann distribution in the plasma. To examine the validity of the Boltzmann distribution, we measured the emission intensities of the neutral iron lines at $367.9 \mathrm{~nm}, 373.5 \mathrm{~nm}, 373.7 \mathrm{~nm}, 374.8 \mathrm{~nm}, 375.8 \mathrm{~nm}$, $376.4 \mathrm{~nm}, 381.6 \mathrm{~nm}$ and $382.5 \mathrm{~nm}$ using an iron plate sample. These intensities 
were expressed as a relative emissivities and plotted as a function of $E_{1}$ at a fixed time $t$. The resulting relation confirms the Boltzmann distribution except for deviations found at the low-energy ends, which are probably due to the lower atom density in the plasma at a reduced air pressure. ${ }^{32}$ We have thus demonstrated that the plasma at reduced gas pressure did satisfy to a good extent the Boltzmann distribution assumption. In this experiment, the emission intensities employed for that purpose are those of the $\mathrm{Cu}$ I $521.8 \mathrm{~nm}$ and $\mathrm{Cu}$ I $510.5 \mathrm{~nm}$ spectral lines. From Fig. 2 and Fig. 3, it should be noted that the ratio between $\mathrm{Cu}$ I $521.8 \mathrm{~nm}$ and $\mathrm{Cu} \mathrm{I} 510.5 \mathrm{~nm}$ is quite the same for the case of LISPS and LIBS for both the time- resolved and time-integrated measurements, leading to a temperature of around 8,000 K. However, in the case of helium at 35 Torr, the plasma temperature was around $10,000 \mathrm{~K}$. This difference is closely related to the propagation speed of the atoms coming out from the primary plasma.

Table 1 shows the comparison between the sensitive emission lines of the tested elements using the arc method, LISPS at air pressure 5 Torr, LISPS at helium pressure 35 Torr, LIBS at air pressure 760 Torr.

\begin{tabular}{|l|c|c|c|c|}
\hline $\begin{array}{l}\text { Sensitive } \\
\text { Emission Line }\end{array}$ & ARC. & $\begin{array}{l}\text { LISPS, air } \\
\text { 5 Torr }\end{array}$ & $\begin{array}{l}\text { LISPS, helium } \\
\text { 35 Torr }\end{array}$ & $\begin{array}{l}\text { LIBS, air 760 } \\
\text { Torr }\end{array}$ \\
\hline Cu I 521,8 nm & 700 & 6100 & 11593 & 2747 \\
\hline Cu I 515,3 nm & 600 & 3536 & 6273 & 1559 \\
\hline Cu I 510,5 nm & 500 & 3198 & 3701 & 981 \\
\hline Cu I 327,4 nm & 3000 & 2186 & 3089 & 2055 \\
\hline Cu I 324,7 nm & 5000 & 2907 & 3673 & 3226 \\
\hline Zn I 636,2 nm & 1000 & 72 & 69 & 100 \\
\hline Zn I 481,0 nm & 400 & 981 & 800 & 605 \\
\hline Zn I 472,2 nm & 400 & 753 & 582 & 485 \\
\hline Zn I 468,0 nm & 300 & 310 & 224 & 264 \\
\hline Ni I 349,5 nm & 1000 & 312 & 237 & 170 \\
\hline Ni I 341,4 nm & 1000 & 255 & 237 & 2463 \\
\hline Ga I 417,2 nm & 2000 & 900 & 1022 & 1797 \\
\hline Ga I 403,2 nm & 1000 & 650 & 811 & 394 \\
\hline Ag I 328,0 nm & 2000 & 276 & 392 & 309 \\
\hline Ag I 338,2 nm & 1000 & 700 & 221 & 112 \\
\hline Ag I 546,5 nm & 1000 & 397 & 330 & 235 \\
\hline Si I 288,1 nm & 500 & 1853 & 1714 & 280 \\
\hline Fe I 373,7 nm & 1000 & 76 & 287 & 17 \\
\hline Au I 242,7 nm & 400 & 28 & & 133 \\
\hline
\end{tabular}

Table 1 Comparison of the emission lines intensity on several targets using arc method, LISPS at air pressure 5 Torr, LISPS at helium pressure 35 Torr, LIBS at air pressure 760 Torr. 
It must be noted that the intensity comparison is only qualitative in nature; for example using the arc method, the first three sensitive emission lines are $\mathrm{Cu}$ I $521.8 \mathrm{~nm}, \mathrm{Cu}$ I $515.3 \mathrm{~nm}$ and $\mathrm{Cu}$ I 510,5 nm, respectively, in the order of decreasing emission intensity. A similar trend is also found in the detection of the same three lines using the LISPS method at air pressure 5 Torr, LISPS at helium pressure 35 Torr, and LIBS method. This shows that there exists a correlation between the sensitive emission lines obtained using both the laser and non laser methods. Specific to the $\mathrm{Cu}$ element, it is worth mentioning that the emission intensity correlation is also observed at the resonant emission lines of $\mathrm{Cu}$, namely $\mathrm{Cu}$ I $327.4 \mathrm{~nm}$ dan $\mathrm{Cu}$ I $324.7 \mathrm{~nm}$. This is extremely important as almost all identification procedures for $\mathrm{Cu}$ uses these two resonant lines, which has a relatively large emission line intensities albeit smaller than the three basic emission lines of $\mathrm{Cu}$ in the green colour region.

For Zn, the basic emission lines of Zn I 481.0 nm, Zn I 472.2 nm and Zn I 468.0 $\mathrm{nm}$ are used. Specific to $\mathrm{Zn}$, the emission intensities of $\mathrm{Zn} \mathrm{I} 481.0 \mathrm{~nm}$ and $\mathrm{Zn} \mathrm{I}$ $472.2 \mathrm{~nm}$ using the arc method are exactly the same. However, using the laser method - LISPS air pressure 5 Torr, LISPS helium 35 Torr and LIBS air pressure 760 Torr - the emission line intensity of $\mathrm{Zn} \mathrm{I} 472.2 \mathrm{~nm}$ is smaller than that of Zn I $481.0 \mathrm{~nm}$. This discrepancy is closely related to the difference in the boiling points; Zinc has a relatively low boiling point $(<1300 \mathrm{~K})$ whereas $\mathrm{Cu}$ has a relatively high boiling point $(<3000 \mathrm{~K})$. It is important to note that the aforementioned explanation is strictly qualitative and only presents a rough picture, which requires further investigations to verify. This phenomenon is also observed for Zn I $468.0 \mathrm{~nm}$. Using the arc discharge method, the emission intensity of Zn I $468.0 \mathrm{~nm}$ is roughly equivalent to $75 \%$ of the emission intensity of Zn I $481.0 \mathrm{~nm}$, whereas using the laser methods, the emission intensity of Zn I $468.0 \mathrm{~nm}$ is only roughly equivalent to $30 \%$ of the emission intensity of Zn I $481.0 \mathrm{~nm}$. This difference in emission intensity of the element $\mathrm{Zn}$ using the arc discharge and laser ablation methods can be clearly observed when identifying Zn using its infra red emission line, Zn I $636.2 \mathrm{~nm}$. Using the arc discharge method, the emission intensity of Zn I $636.2 \mathrm{~nm}$ is approximately 2.5 times the intensity of $\mathrm{Zn}$ I $481.0 \mathrm{~nm}$, whereas using the laser methods, the emission intensity of Zn I $636.2 \mathrm{~nm}$ is almost 10 times smaller than the intensity of Zn I $481.0 \mathrm{~nm}$. Specifically for the emission line of Zn I $636.2 \mathrm{~nm}$, a simple explanation could be found in the relatively large excitation energy of Zn I $636.2 \mathrm{~nm}$ which is around $7.7 \mathrm{eV}$. Some verification of this hypothesis was provided by raising the laser energy to $200 \mathrm{~mJ}$, which resulted in the enhancement of emission intensity of $\mathrm{Zn} \mathrm{I} 636.2 \mathrm{~nm}$ to the level close to emission intensity of Zn I $481.0 \mathrm{~nm}$. However, further study is still underway for more thorough explanation. 
Using the arc discharge technique for the element Ni, emission lines Ni I 349.3 $\mathrm{nm}$ and Ni I $341.4 \mathrm{~nm}$, have exactly the same intensities. A similar trend is observed using the LISPS method in helium at 35 Torr. However, the LISPS method in air at 5 Torr and LIBS in air at 760 Torr, produced slight differences in the emission intensities of the two emission lines.

For the element Ga, the emission lines chosen are Ga I $417.2 \mathrm{~nm}$ and Ga I 403.2 $\mathrm{nm}$. Using the arc discharge technique, it is clearly observed that the emission intensity of Ga I $417.2 \mathrm{~nm}$ is twice that of Ga I $403.2 \mathrm{~nm}$. Measurements with the laser methods yielded a result showing an emission intensity of Ga I 417.2 $\mathrm{nm}$ higher than that of Ga I 403.2 which is still qualitatively consistent with result given by arc discharge measurement.

Further identification of Ag was also carried out and three prominent Ag lines was selected, Ag I $328.0 \mathrm{~nm}$, Ag I $338.2 \mathrm{~nm}$ and Ag I $546.5 \mathrm{~nm}$. It should be noted that there is a lack of consistent relationship between emission intensities obtained using laser techniques and those obtained by the arc discharge for the above Ag emission lines. However, ones should not jump to conclude in this stage that laser techniques are inferior compared to arc discharge, because this study was carried out in order to mapping the sensitive emission lines using laser techniques.

Turning to Si emission line, we chose Si I $288.1 \mathrm{~nm}$ in this study because the other three prominent silicone lines, Si I $250.6 \mathrm{~nm}$, Si I $251.6 \mathrm{~nm}$ dan Si I 252.6 $\mathrm{nm}$ could not be resolved using our spectrograph due to the relatively weak emission intensity in this region without employing wider opening of the spectrograph's slit width. However, it should be noted that significant difference in emission intensity was found even between the LISPS and LIBS results. This discrepancy is probably due to the short life time of the excited state associated with the Si I $288.1 \mathrm{~nm}$ emission line, and as a result the peak of the emission intensity would be attained before 200 ns. In LIBS, the minimum gateable signal is obtained at 200 ns when the aforementioned emission intensity has already vanished. The opposite case observed on the Au I $242.7 \mathrm{~nm}$ emission line remained unexplained at this stage of the study.

Clarification and interpretation regarding the Fe I $373.7 \mathrm{~nm}$ emission line is not given in this paper due to inadequate spectral resolution used in this study. However, a comparison with the arc discharge result indicate that the laser techniques seem to offer a better choice for spectrochemical analysis.

Table 2 showed the emission intensity stability attained by the laser techniques. It is useful for comparison to note that the stability of the well established nonlaser technique usually ranges between 5 to $20 \%$. The results obtained in this 
study in most cases are better than non-laser technique. However, for some elements, the emission intensity stabilities in the case of LISPS using air at 5 Torr and LISPS using helium at 35 Torr are very poor, for instance for Au and Fe. These poor performance have their origin in the extremely thin samples $(0.1$ $\mathrm{mm}$ ) as compared to $0.4 \mathrm{~mm}$ for other samples. This resulted in serious inhomogeneous surfaces of the samples, which in turn gave rise to fluctuation of the lens to sample distance (LSD) and hence the fluctuation of laser power density arriving at the sample surface. We have found in a separate experiment using ordinary iron plate of $10 \mathrm{~mm}$ thickness, that the stability for the same iron line was readily improved to $3.01 \%$ in the case of air at 5 Torr and $2.88 \%$ in the case of helium at 35 Torr. The emission stability of this new technique can be improved in the near future by using high quality motorized LSD device along with high stability samples holder used to rotate the samples during all of the experiments.

\begin{tabular}{|c|c|c|c|c|c|c|c|c|c|}
\hline \multirow{2}{*}{$\begin{array}{l}\text { Sensitive } \\
\text { Emission } \\
\text { Lines (A) }\end{array}$} & \multicolumn{3}{|c|}{ LISPS, air 5 Torr } & \multicolumn{3}{|c|}{ LISPS, helium 35 Torr } & \multicolumn{3}{|c|}{ LIBS, air 760 Torr } \\
\hline & $\mathrm{x}$ & $\sigma$ & $\frac{\sigma}{x} \times 100 \%$ & $\mathrm{X}$ & $\sigma$ & $\frac{\sigma}{x} \times 100 \%$ & $\mathrm{x}$ & $\sigma$ & $\frac{\sigma}{x} \times 100 \%$ \\
\hline Cu I 5218 & 6099 & 305 & $5 \%$ & 11593 & 899 & $7,75 \%$ & 2747 & 587 & $21,37 \%$ \\
\hline 5153 & 3536 & 180 & $5,09 \%$ & 6273 & 539 & $8,59 \%$ & 1559 & 337 & $21,62 \%$ \\
\hline 5105 & 3198 & 165 & $5,16 \%$ & 3702 & 291 & $7,86 \%$ & 981 & 180 & $18,35 \%$ \\
\hline 3274 & 2186 & 108 & $4,94 \%$ & 3089 & 216 & $6,99 \%$ & 2055 & 299 & $14,55 \%$ \\
\hline 3247 & 2907 & 180 & $6,19 \%$ & 3673 & 260 & $7,08 \%$ & 3226 & 418 & $12,96 \%$ \\
\hline Zn I 6362 & 72 & 18 & $25 \%$ & 69 & 11 & $15,94 \%$ & 100 & 14 & $14 \%$ \\
\hline 4810 & 981 & 62 & $6,32 \%$ & 800 & 45 & $5,63 \%$ & 605 & 95 & $15,7 \%$ \\
\hline 4722 & 753 & 49 & $6,51 \%$ & 582 & 41 & $7,04 \%$ & 485 & 67 & $13,81 \%$ \\
\hline 4680 & 310 & 22 & $7,1 \%$ & 224 & 30 & $13,39 \%$ & 264 & 41 & $15,53 \%$ \\
\hline Ni I 3495 & 312 & 38 & $12,18 \%$ & 237 & 28 & $11,81 \%$ & 213 & 32 & $15,02 \%$ \\
\hline 3414 & 255 & 47 & $18,43 \%$ & 237 & 16 & $6,75 \%$ & 170 & 17 & $10 \%$ \\
\hline Ga I 4172 & 900 & 39 & $4,33 \%$ & 1022 & 40 & $3,91 \%$ & 2463 & 226 & $9,18 \%$ \\
\hline 4032 & 650 & 43 & $6,62 \%$ & 811 & 69 & $8,51 \%$ & 1797 & 170 & $9,46 \%$ \\
\hline Ag 3280 & 276 & 25 & $9,06 \%$ & 392 & 38 & $9,69 \%$ & 394 & 101 & $25,63 \%$ \\
\hline 3382 & 221 & 26 & $11,76 \%$ & 309 & 24 & $7,77 \%$ & 277 & 78 & $28,16 \%$ \\
\hline 5465 & 397 & 52 & $13,1 \%$ & 330 & 43 & $13,03 \%$ & 112 & 28 & $25 \%$ \\
\hline 2881 & 1853 & 52 & $2,81 \%$ & 1714 & 60 & $2,08 \%$ & 235 & 89 & $37,87 \%$ \\
\hline $\mathrm{Fe} \quad 3737$ & 76 & 15 & $19,74 \%$ & 287 & 23 & $8,01 \%$ & 133 & 44 & $33,08 \%$ \\
\hline $\mathrm{Au} \quad 2427$ & 28 & 7 & $25 \%$ & 17 & 7 & $41,18 \%$ & 280 & 49 & $17,5 \%$ \\
\hline
\end{tabular}

Table 2 Average emission intensities and deviation standard of sensitive emission lines of several metals using laser techniques. 
We note further from Table 2, that LISPS in air at 5 Torr and in helium at 35 Torr yielded better stability than LIBS. This is mainly related to the complicated interaction taking place in the high density plasma generated at 760 Torr.

\section{Conclusion}

A comparative study has been undertaken on the sensitive emission intensity of a few metal samples using non laser and laser methods. The results obtained indicate that there exists a systematic correlation between the results obtained by the laser methods for a number of metal samples. It was shown that in some cases where such a correlation did not feature as solidly, this discrepancies were likely to have their origin in the specific experimental condition affecting the excitation processes. Further, the standard deviation measurement results from the two laser methods shows that the new LISPS technique developed in our laboratory is inherently superior to the LIBS technique now commonly used as the standard method of spectrochemical analysis using laser technique.

\section{References}

1. Harrison, G. R., in Massachusetts Institute of Technology Wavelength Tables, The M.I.T. Press, Cambridge, Massachusetts, and London, England, 1939 ed.

2. $\quad$ Phelps III, F. M., in Massachusetts Institute of Technology Wavelength Tables II, The M.I.T. Press, Cambridge, Massachusetts, and London, England, 1969 ed.

3. $\quad$ Brech, F. \& Cross, L., Appl. Spectrosc. 16 (1962) 59.

4. Laqua, K., in Analytical Laser Spectroscopy, N. Omenetto, Ed., Wiley, New York, 1979), p. 47.

5. Piepmeier, E. H., in Analytical Applications of Lasers, Wiley, New York, 1986, p.627.

6. Cremers, D. A. \& Radziemski, L. J., in Laser Spectroscopy and its Applications, R.W. Solarz and J.A. Paisner (Ed), Marcel Dekker, New York, 1987, p. 351.

7. Loree, T. R. \& Radziemski, L. J., Plasma Chem. And Plasma Proc. 1, 271 (1981).

8. Radziemski, L. J. \& Loree, T. R., Plasma Chem. And Plasma Proc. 1, 281, (1981).

9. $\quad$ Cremers, D. A., Appl. Spectrosc. 41, 572 (1987).

10. Cremers, D. A., Anal. Chem. 55, 1252 (1983).

11. Cremers, D.A., Appl. Spectrosc. 38, 721 (1984).

12. Radziemski, L. J. \& Cremers, D. A., in Laser Induced Plasma and Applications, D.A. Cremers (Ed), Marcel Dekker, New York, 1989, pp. 295-346. 
13. Piepmeier, E.H. \& Osten, D.E., Appl. Spectrosc. 25, 642 (1971).

14. Dimitrov, G. \& Gagov, V., Spectrosc. Lett. 10, 337 (1979).

15. Gibson, A. F., Hughes, T. P. \& Ireland, C. L. M., J. Phys. D: Appl. Phys. 4, 1527 (1971).

16. Kuzuya, M. \& Mikami, O., Jpn. J. Appl. Phys. 29, 1568 (1990).

17. Iida, Y., Spectrochim. Acta. 45B, 1353 (1990).

18. Leis, F., Sdorra, W., Ko, J. B. \& Niemax, K., Microchim. Acta II, 185 (1989).

19. Lee, Y. I., Thiem, T. L., Kim, G. H., Teng, Y. Y. \& Sneddon, J., Appl. Spectrosc. 46, 1597 (1992).

20. Wu, J. D., Pan, Q. \& Chen, S. C., Appl. Spectrosc. 51, 883 (1997).

21. Kagawa, K., Matsuda, Y., Yokoi, S. \& Nakajima, S., J. Anal. Atom. Spectrom. 3, 415 (1988).

22. Kagawa, K., Yanagihara, S. \& Yokoi, S., J. Spectrosc. Soc. Jap. 34, 306 (1985).

23. Kagawa, K. \& Kurniawan, H., Proc. Int. Work Shop on Strong Shock Waves (Chiba University Press, 1991).

24. Kagawa, K., Kawai, K., Tani, M. \& Kobayashi, T., Appl. Spectrosc. 48, 198 (1994).

25. Kagawa, K., Hattori, H., Ishikane, M., Ueda, M. \& Kurniawan, H., Anal. Chim. Acta, 299, 393 (1995).

26. Kurniawan, H., Nakajima, S., Batubara, J. E., Marpaung, A. M., Okamoto, M. \& Kagawa, K., Appl. Spectrosc. 49, 1067 (1995).

27. Kurniawan, H., Kagawa, K., Okamoto, M., Ueda, M., Kobayashi, T. \& Nakajima, S., Appl. Spectrosc. 50, 299 (1996).

28. Kurniawan, H., Ishikawa, Y., Nakajima, S. \& Kagawa, K., Appl. Spectrosc. 51, 1769 (1997).

29. Suliyanti, M. M., Hedwig, R., Kurniawan, H. \& Kagawa, K., Jpn. J. Appl. Phys. 37, 146 (1998).

30. Kurniawan, H., Pardede, M., Kagawa, K. and Tjia, M.O., J. Spectrosc. Soc. Jap. 47, 5, 220 (1998).

31. Kurniawan, H., Setia Budi, W., Suliyanti, M. M., Marpaung, A. M. \& Kagawa, K., J. Phys. D: Appl. Phys. 30, 3335 (1997).

32. Setia Budi, W., Suyanto, H., Kurniawan, H., Tjia, M.O. \& Kagawa, K., Appl. Spectrosc. 53, 6, 719 (1999).

33. Setia Budi, W., Baskoro, W. T., Pardede, M., Kurniawan, H., Tjia, M. O. \& Kagawa, K., Appl. Spectrosc. 53, 11, 1347 (1999).

34. Setia Budi, W., Rahman, A., Kurniawan, H., Tjia, M. O. \& Kagawa, K., Rev. of Laser Eng. 29, 3, 180 (2001).

35. Kurniawan, H., Suliyanti, M. M., Lie, T. J., Kagawa, K. \& Tjia, M. O., Spectrochim. Acta Part B: At. Spectrom. B56, 9, 1407 (2001).

36. Kurniawan, H., Lahna, K., Lie, T. J., Kagawa, K. \& Tjia, M. O., Appl. Spectrosc. 55, 1, 92 (2001). 
37. Ready, J. F., Effect of High Power Laser Radiation (Academic Press, New York, 1971). (a) adopted from A.M. Marpaung, doctoral thesis, University of Indonesia, 2001).

38. Hughes, T. P., Plasma and Laser Light (Adam Hilger, Bristol, 1975).

39. Cremers, D. A., Radziemski, L. J., Laser Plasmas for Chemical Analysis, in Laser Spectroscopy and Its Applications, L.J. Radziemski, R.W. Solarz and J.A. Paisner, Eds. (Marcel Dekker, New York, 1979). chapter 5.

40. Weyl, G. M., Physics of Laser-Induced Breakdown: An Update, in Laser Induced Plasmas and Applications, L.J. Radziemski and D.A. Cremers, Eds. (Marcel Dekker, New York, 1989) chapter 1.

41. Scott, R. \& Strasheim, A., Spectrochim. Acta, B25, 311 (1970).

42. Lewis, A. L., Beenen, G. J., Hasck, J. W. \& Piepmeier, E. H., Appl. Spectrosc. 37, 263 (1983).

43. Goldsack, T. J., Kilkenny, J. D., McGowan, B. J., Veats, S. A., Key, M. H., Rumsby, P. T., Toner, W. T., Opt. Commun. 42, 55 (1982).

44. Ni, S. W., Lu, J., He, A. Z., Ma, Z., Zhon, J. L., Opt. Commun. 74, 185 (1989).

45. Lee, Y. I., Sneddon, J., Microchem. J. 50, 235 (1994).

46. Hwang, Z. W., Teng, Y. Y., Li, K. P. \& Sneddon, J., Appl. Spectrosc. 45, 435 (1991).

47. Root, R. G., Modelling of Post Breakdown Phenomena, in Laser Induced Plasmas and Applications, L.J. Radziemski and D.A. Cremers, Eds (Marcel Dekker, New York, 1989) chapter 2.

48. Mao, X. L., Chan, W. T., Shannon, M., Russso, R. E., J. Appl. Phys. 74, 4915 (1993).

49. Kurniawan, H., Tjia, M. O., Barmawi, M., Yokoi, S., Kimura, Y. \& Kagawa, K., J. Phys. D: Appl. Phys. 28, 879 (1995).

50. Kurniawan, H., Kobayashi, T. \& Kagawa, K., Appl. Spectrosc. 46, 581 (1992).

51. Sedov, L. I., Similarity and Dimensional Methods in Mechanics (Academic Press, New York, London, 1959) p.213. 